\title{
On a Numerical Radius Preserving Onto Isometry on $\mathscr{L}(X)$
}

\author{
Sun Kwang Kim \\ Department of Mathematics, Kyonggi University, Suwon 443-760, Republic of Korea \\ Correspondence should be addressed to Sun Kwang Kim; sunkwang@kgu.ac.kr
}

Received 17 August 2016; Accepted 25 September 2016

Academic Editor: Ajda Fošner

Copyright (C) 2016 Sun Kwang Kim. This is an open access article distributed under the Creative Commons Attribution License, which permits unrestricted use, distribution, and reproduction in any medium, provided the original work is properly cited.

We study a numerical radius preserving onto isometry on $\mathscr{L}(X)$. As a main result, when $X$ is a complex Banach space having both uniform smoothness and uniform convexity, we show that an onto isometry $T$ on $\mathscr{L}(X)$ is numerical radius preserving if and only if there exists a scalar $c_{T}$ of modulus 1 such that $c_{T} T$ is numerical range preserving. The examples of such spaces are Hilbert space and $L_{p}$ spaces for $1<p<\infty$.

\section{Introduction}

In this paper, we study a numerical radius preserving onto isometry on the set of bounded linear operators. We begin with some notation to present its definition. Let $X$ be a Banach space over the field $\mathbb{K}=\mathbb{R}$ or $\mathbb{C}$. We use $X^{*}$ and $\mathscr{L}(X)$ for the dual of $X$ and the space of bounded linear operators from $X$ to $X$, respectively. We denote by $B_{X}$ (resp., $S_{X}$ ) the closed unit ball of $X$ (resp., the unit sphere of $X$ ).

The numerical radius and numerical range of an operator $A \in \mathscr{L}(X)$ are given by

$$
\begin{aligned}
v(A) & =\sup \left\{\left|x^{*}(A x)\right|:\left(x, x^{*}\right) \in \Pi(X)\right\}, \\
V(A) & =\overline{\operatorname{conv}\left\{x^{*}(A x):\left(x, x^{*}\right) \in \Pi(X)\right\}},
\end{aligned}
$$

where $\Pi(X)=\left\{\left(x, x^{*}\right) \in S_{X} \times S_{X^{*}}: x^{*}(x)=1\right\}$.

Note that there are many different definitions of numerical range. The above definition of $V(A)$ is sometimes called Banach algebra numerical range to distinguish from others. It is worth mentioning that it is also usual to study spatial numerical range $\left\{x^{*}(A x):\left(x, x^{*}\right) \in \Pi(X)\right\}$. From the definition, we see that $v(A) \leq\|A\|$ for every $A \in \mathscr{L}(X)$.

The concept of numerical range was introduced by $\mathrm{G}$. Lumer and F. Bauer in the sixties. Later this was extended to arbitrary continuous functions on a unit sphere of Banach spaces. On the other hand, the study of numerical radius was initiated by Harris for polynomials and holomorphic functions. We can find more details in [1-3].
There has been many different types of research on these concepts. Among them, we can find a lot of works on linear maps which preserve numerical radius or numerical range [4].

We say that an operator $T$ on $\mathscr{L}(X)$ preserves numerical radius (numerical range) when

$$
\begin{gathered}
v(T(A))=v(A) \\
(V(T(A))=V(A))
\end{gathered}
$$

for every $A \in \mathscr{L}(X)$. It is clear that every numerical range preserving map is numerical radius preserving.

In 1975, Pellegrini [5] studied numerical range preserving operators on a Banach algebra. Particularly, when $\mathscr{H}$ is a complex Hilbert space, it was shown that an isomorphism $T$ on $\mathscr{L}(\mathscr{H})$ is $C^{*}$-isomorphism if and only if it is numerical range preserving. Later, Chan [6] showed that an isomorphism $T$ on $\mathscr{L}(\mathscr{H})$ is numerical radius preserving if and only if $c_{T} T$ is a $C^{*}$-isomorphism for some scalar $c_{T}$ of modulus 1 . These results say that for each numerical radius preserving isomorphism $T$ on $\mathscr{L}(\mathscr{H})$ there exists a scalar $\mathcal{c}_{T}$ of modulus 1 such that $c_{T} T$ is a numerical range preserving mapping.

In Section 2, we deduce a similar result for an onto isometry when a complex Banach space $X$ has both uniform convexity and uniform smoothness. Indeed, we show that if $T$ is a numerical radius preserving onto isometry on $\mathscr{L}(X)$ then there exists a scalar $c_{T}$ of modulus 1 such that $c_{T} T$ is numerical range preserving. It is known that Hilbert space and $L_{p}$ for $1<p<\infty$ have both uniform convexity and 
uniform smoothness. However, we see that this does not hold when $X=\ell_{\infty}^{2}$.

\section{Results}

We first recall the definition of uniform convexity.

For every $\epsilon \in(0,2]$, the modulus of convexity of a Banach space $(X,\|\cdot\|)$ is defined by

$$
\delta(\epsilon)=\inf \left\{1-\left\|\frac{x+y}{2}\right\|: x, y \in B_{X},\|x-y\| \geq \epsilon\right\} .
$$

A Banach space $(X,\|\cdot\|)$ is said to be uniformly convex if $\delta(\epsilon)>0$ for all $\epsilon \in(0,2]$.

It is well known that every uniformly convex space is strictly convex and $L_{p}$ is uniformly convex when $1<p<\infty$.

Very recently, the following characterization of uniform convexity was shown [7].

Theorem 1. A Banach space $X$ is uniformly convex if and only if for each $\varepsilon>0$ there is $\eta(\varepsilon)>0$ such that for any $\left(x, x^{*}\right) \in$ $S_{X} \times S_{X^{*}}$ with $\left|x^{*}(x)\right|>1-\eta(\varepsilon)$ there exists $y \in S_{Y}$ satisfying

$$
\begin{aligned}
x^{*}(y) & =1, \\
\|y-x\| & <\varepsilon .
\end{aligned}
$$

From this theorem, we show the following lemma.

Lemma 2. Let $X$ be a uniformly convex space. For any $\varepsilon>0$ there exists $0<\beta(\varepsilon)<\varepsilon$ such that if two pairs $\left(x, x^{*}\right),\left(y, y^{*}\right) \in$ $\Pi(X)$ satisfy $\left\|x^{*}-y^{*}\right\|<\beta(\varepsilon)$ then $\|x-y\|<\varepsilon$.

Proof. Let $\eta(\cdot)$ be the function in Theorem 1 and assume that $\left(x, x^{*}\right),\left(y, y^{*}\right) \in \Pi(X)$ satisfy $\left\|x^{*}-y^{*}\right\|<\eta(\varepsilon)$.

Note that $x$ is the unique element in $B_{X}$ such that $x^{*}(x)=$ 1 by the strict convexity of $X$. Since $\left|x^{*}(y)\right| \geq\left|y^{*}(y)\right|-\mid\left(y^{*}-\right.$ $\left.x^{*}\right)(x) \mid>1-\eta(\varepsilon)$, we see that $\|x-y\|<\varepsilon$ from Theorem 1 . Hence we may take $\beta(\varepsilon)=\min \{\eta(\varepsilon), \varepsilon / 2\}$.

It is worth remarking that the converse of Lemma 2 is true when the space $X$ is finite dimensional. Indeed, if $X$ is not uniformly convex, then there exist $\varepsilon>0$ and a sequence of pairs $\left(x_{i}, y_{i}\right) \in B_{X} \times B_{X}$ such that $\left\|x_{i}-y_{i}\right\|>\varepsilon$ and $\| x_{i}+$ $y_{i} \|$ converge to 2 . Since $B_{X}$ is compact, we may assume that $\left(x_{i}\right)$ and $\left(y_{i}\right)$ converge to $x$ and $y$, respectively. It is clear that $\|x+y\|=2$ and so there exists a functional $f \in S_{X^{*}}$ such that $f(x+y)=2$. Since $(x, f),(y, f) \in \Pi(X)$ and $\|x-y\|>\varepsilon$, we get the desired contradiction.

In paper [6] of Chan, it was shown that if $T$ is a numerical radius preserving isomorphism on $\mathscr{L}(\mathscr{H})$, then $T(I)=c_{T} I$ for some constant $c_{T}$ of modulus 1 , where $I$ is the identity map on $\mathscr{H}$. We show that the same result holds for an onto isometry on $\mathscr{L}(X)$ when $X$ has uniform convexity and uniform smoothness. Before that, we first see the following.

Lemma 3. If $T$ is a numerical radius preserving map on $\mathscr{L}(X)$, then for each $A \in \mathscr{L}(X)$ there exists a scalar $\alpha_{A}$ of modulus 1 such that $v(T(I))+v(T(A))=v\left(T(I)+\alpha_{A} T(A)\right)$.

Proof. We first see that for any $A \in \mathscr{L}(X)$ there is a scalar $\alpha_{A}$ of modulus 1 satisfying $v\left(I+\alpha_{A} A\right)=1+v(A)$. Indeed, for a sequence $\left(x_{i}, x_{i}^{*}\right) \in \Pi(X)$ such that $\left|x_{i}^{*}\left(A x_{i}\right)\right|$ converges to $v(A)$, we may assume that $x_{i}^{*}\left(A x_{i}\right)$ converges to some constant $\beta$. Let $\alpha_{A}=\bar{\beta} /|\beta|$. Then, we have that $x_{i}^{*}\left(\left(I+\alpha_{A} A\right) x_{i}\right)$ converges to $1+|\beta|=1+v(A)$. Since it is clear that $v\left(I+\alpha_{A} A\right) \leq$ $1+v(A)$, we deduce $v\left(I+\alpha_{A} A\right)=1+v(A)$.

The fact that $T$ preserves numerical radius gives $v(T(I))+$ $v(T(A))=v(I)+v(A)=v\left(I+\alpha_{A} A\right)=v\left(T\left(I+\alpha_{A} A\right)\right)=$ $v\left(T(I)+\alpha_{A} T(A)\right)$.

Lemma 4. Assume $X$ is a strictly convex space and an operator $A \in \mathscr{L}(X)$ satisfies $\|A\|=1$. If absolute value of every element in $V(A)$ is 1 , then $A=c_{A} I$ for some constant $c_{A}$ of modulus 1 .

Proof. From the assumption, we see that $\|A x\|=1=$ $\left|x^{*}(A x)\right|$ for arbitrary $x \in S_{X}$ and $x^{*}$ satisfying $x^{*}(x)=1$. Since $X$ is strictly convex, $x^{*}$ attains its norm only at $\{\lambda x$ : $|\lambda|=1\}$. This implies that $A x=\lambda_{x} x$ for some constant $\lambda_{x}$ of modulus 1 .

Now assume that there exist two elements $x_{1}, x_{2} \in S_{X}$ such that $\lambda_{x_{1}} \neq \lambda_{x_{2}}$. Note that $x_{1}$ and $x_{2}$ are linearly independent. Consider $z=\left(x_{1}+x_{2}\right) /\left\|x_{1}+x_{2}\right\|$ and let $A z=\lambda_{z} z$. This means that

$$
\frac{\lambda_{z} x_{1}+\lambda_{z} x_{2}}{\left\|x_{1}+x_{2}\right\|}=\lambda_{z} z=A_{z}=\frac{\lambda_{x_{1}} x_{1}+\lambda_{x_{2}} x_{2}}{\left\|x_{1}+x_{2}\right\|} .
$$

Hence, we have $\left(\lambda_{z}-\lambda_{x_{1}}\right) x_{1}=\left(\lambda_{x_{2}}-\lambda_{z}\right) x_{2}$ which is a contradiction.

Theorem 5. Assume $X$ has both uniform convexity and uniform smoothness. If $T$ is a numerical radius preserving onto isometry on $\mathscr{L}(X)$, then $T(I)=c_{T} I$ for some constant $c_{T}$ of modulus 1.

Proof. From Lemma 3, for each $A \in \mathscr{L}(X)$, there is $\alpha_{A}$ so that $v(T(I))+v(T(A))=v\left(T(I)+\alpha_{A} T(A)\right)$.

We now show that $\left|x^{*}(T(I)(x))\right|=v(T(I))=v(I)=1$ for any $\left(x, x^{*}\right) \in \Pi(X)$. If this is not true, there exists $\left(z_{0}, z_{0}^{*}\right) \in$ $\Pi(X)$ such that $\left|z_{0}^{*}\left(T(I)\left(z_{0}\right)\right)\right|<1$. Take an operator $B \in$ $\mathscr{L}(X)$ so that $T(B)(x)=z_{0}^{*}(x) z_{0}$ for each $x \in X$ and take $0<\gamma<1$ such that $\left|z_{0}^{*}\left(T(I)\left(z_{0}\right)\right)\right|<\gamma<1$.

Let $\delta>0$ be a number satisfying that every $\left(z, z^{*}\right) \in \Pi(X)$ with $\left\|z-z_{0}\right\|<\delta$ and $\left\|z^{*}-z_{0}^{*}\right\|<\delta$ implies $\left|z^{*}(T(I)) z\right|<\gamma$. Since $X$ is uniformly convex, we may take $\beta(\cdot)$ in Lemma 2 .

For a pair $\left(y, y^{*}\right) \in \Pi(X)$, if $\left\|y^{*}-z_{0}^{*}\right\|<\beta(\delta)<\delta$, then $\left\|y-z_{0}\right\|<\delta$. This gives that

$$
\begin{aligned}
& \left|y^{*}\left(T\left(I+\alpha_{B} B\right)(y)\right)\right| \\
& \quad \leq\left|y^{*}(T(I)(y))\right|+\left|y^{*}(T(B)(y))\right|<\gamma+1 .
\end{aligned}
$$

On the other hand, there exists $\varepsilon>0$ such that if $\left(y, y^{*}\right) \epsilon$ $\Pi(X)$ satisfies $\left\|y^{*}-z_{0}^{*}\right\| \geq \beta(\delta)$, then $\left|y^{*}\left(z_{0}\right)\right| \leq 1-\varepsilon$. To see that this is true, we note that dual of uniformly smooth space is uniformly convex. Hence $X^{*}$ is uniformly convex. We may use again Lemma 2 , and then for some small enough $\varepsilon>0$ if $\left(y, y^{*}\right) \in \Pi(X)$ satisfies $\left|y^{*}\left(z_{0}\right)\right|>1-\varepsilon$ then $\left\|y^{*}-z_{0}^{*}\right\|<\beta(\delta)$. 
Therefore we see that if $\left(y, y^{*}\right) \in \Pi(X)$ satisfies that $\| y^{*}-$ $z_{0}^{*} \| \geq \beta(\delta)$, then $\left|y^{*}(T(B)(y))\right|=\left|y^{*}\left(z_{0}\right) z_{0}^{*}(y)\right| \leq 1-\varepsilon$. We have

$$
\begin{aligned}
& \left|y^{*}\left(T\left(I+\alpha_{B} B\right)(y)\right)\right| \leq\left|y^{*}(T(I)(y))\right|+\left|y^{*} T(B) y\right| \\
& \quad \leq 1+1-\varepsilon .
\end{aligned}
$$

This shows that $v\left(T\left(I+\alpha_{B} B\right)\right) \leq \max \{2-\varepsilon, \gamma+1\}<2=$ $v(T(I))+v(T(B))$ and so we have desired contradiction.

From Lemma 4, we see that $T(I)=c_{T} I$ for some modulus 1 constant $c_{T}$.

Remark 6. From Theorem 5, we can easily construct onto isometries which are not numerical radius preserving. For example, consider 2-dimensional Hilbert space $\mathbb{R}^{2}$; we define an operator $B: \mathbb{R}^{2} \rightarrow \mathbb{R}^{2}$ by $B\left(x_{1}, x_{2}\right)=\left(x_{1},-x_{2}\right)$ for every $\left(x_{1}, x_{2}\right) \in \mathbb{R}^{2}$. It is clear that the operator $T$ on $\mathscr{L}\left(\mathbb{R}^{2}\right)$ given by $T(A)=B \circ A$ for every $A \in \mathscr{L}\left(\mathbb{R}^{2}\right)$ is an onto isometry. However, this does not preserve numerical radius since $T(I) \neq c_{T} I$ for any $c_{T}$.

We denote a state space $\delta$ of a Banach algebra $\mathscr{L}(X)$ by

$$
\mathcal{S}=\left\{f \in \mathscr{L}(X)^{*}:\|f\|=1=f(I)\right\} .
$$

When $X$ is a complex Banach space, according to [5, Theorem 2.2], if the adjoint operator $S^{*}$ of operator $S$ : $\mathscr{L}(X) \rightarrow \mathscr{L}(X)$ satisfies that $S^{*}(\mathcal{S}) \subset \mathcal{S}$, then $V(S(A)) \subset$ $V(A)$. For $T$ in Theorem 5 , since $T_{0}=c_{T}^{-1} T$ is an onto isometry and so satisfies $T_{0}^{*}(\mathcal{S})=\mathcal{S}$, we see that $V\left(T_{0}(A)\right)=V(A)$. Now, we deduce the following main result.

Theorem 7. Assume a complex Banach space $X$ has both uniform convexity and uniform smoothness. An onto isometry $T$ on $\mathscr{L}(X)$ is numerical radius preserving if and only if there exists a scalar $c_{T}$ of modulus 1 such that $c_{T} T$ is a numerical range preserving mapping.

Remark 8. Theorem 7 does not hold for some Banach spaces. In order to see this, let us recall numerical index $n(X)$ of a Banach space.

$$
n(X)=\inf \left\{v(T): T \in S_{\mathscr{L}(X)}\right\} .
$$

We see that $n(X)$ is the greatest constant $k \geq 0$ such that $k\|T\| \leq v(T)$ for every $T \in \mathscr{L}(X)$, and so $v$ is equivalent to the operator norm if and only if $n(X)>0$. For more information, we give [8-11].

From the definition of numerical index, if $n(X)=1$, then every isometry on $\mathscr{L}(X)$ preserves numerical radius. There are many classes of Banach spaces having numerical index 1, like $c_{0}, \ell_{1}$, and $\ell_{\infty}$ [12]. Among them, one of the simplest examples having numerical index 1 is $\mathbb{R}^{2}$ as the typical subspace of $\ell_{\infty}$. Since the same operator $T$ defined in Remark 6 is also an onto isometry on this space, $T$ preserves numerical radius. However, this does not preserve numerical range. The reason is that we have $V(I)=\{1\}$ and $-1=$ $(0,1) T(I)(0,1) \in V(T(I))$.
Remark 9. It is not possible to say that Theorem 7 is true for every isometry. Consider shift operators $B_{1}$ and $B_{2}$ on $\ell_{p}(1<$ $p<\infty)$ defined by $B_{1}\left(\left(x_{i}\right)_{i=1}^{\infty}\right)=\left(x_{i}\right)_{i=2}^{\infty}$ and $B_{2}\left(\left(x_{i}\right)_{i=1}^{\infty}\right)=$ $\left(x_{i}\right)_{i=0}^{\infty}$ for $\left(x_{i}\right)_{i=1}^{\infty} \in \ell_{p}$, where $x_{0}=0$. Then, it is easy to see that an operator $T$ given by $T(A)=B_{2} \circ A \circ B_{1}$ for each $A \epsilon$ $L\left(\ell_{p}\right)$ is a numerical radius preserving isometry. However, $T$ does not preserve numerical range since $V(I)=\{1\}$ and $0=$ $(1,0,0,0, \ldots) T(I)(1,0,0,0, \ldots) \in V(T(I))$.

\section{Competing Interests}

The author declares that there is no conflict of interests regarding the publication of this paper.

\section{Acknowledgments}

This work was supported by Kyonggi University Research Grant 2015.

\section{References}

[1] F. F. Bonsall and J. Duncan, Numerical Ranges I, vol. 2 of Lecture Notes in Mathematics, London Mathematical Society, Cambridge, UK, 1971.

[2] F. F. Bonsall and J. Duncan, Numerical Ranges II, London Mathematical Society Lecture Note Series 10, Cambridge University Press, Cambridge, Uk, 1973.

[3] L. A. Harris, "The numerical range of holomorphic functions in Banach spaces," The American Journal of Mathematics, vol. 93, pp. 1005-1019, 1971.

[4] C.-K. Li, "A survey on linear preservers of numerical ranges and radii," Taiwanese Journal of Mathematics, vol. 5, no. 3, pp. 477496, 2001.

[5] V. J. Pellegrini, "Numerical range preserving operators on a Banach algebra," Polska Akademia Nauk. Instytut Matematyczny. Studia Mathematica, vol. 54, no. 2, pp. 143-147, 1975.

[6] J.-T. Chan, "Numerical radius perserving operators on $\mathrm{B}(\mathrm{H})$," Proceedings of the American Mathematical Society, vol. 123, no. 5, pp. 1437-1439, 1995.

[7] S. K. Kim and H. J. Lee, "Uniform convexity and Bishop-PhelpsBollobás property," Canadian Journal of Mathematics, vol. 66, no. 2, pp. 373-386, 2014.

[8] K. Boyko, V. Kadets, M. Martín, and D. Werner, "Numerical index of Banach spaces and duality," Mathematical Proceedings of the Cambridge Philosophical Society, vol. 142, no. 1, pp. 93-102, 2007.

[9] C. Finet, M. Martín, and R. Payá, "Numerical index and renorming," Proceedings of the American Mathematical Society, vol. 131, no. 3, pp. 871-877, 2003.

[10] V. Kadets, M. Martn, and R. Payá, "Recent progress and open questions on the numerical index of Banach spaces," Revista de la Real Academia de Ciencias Exactas, Físicas y Naturales, Serie A: Matemáticas, vol. 100, pp. 155-182, 2006.

[11] M. Martín, J. Merí, M. Popov, and B. Randrianantoanina, "Numerical index of absolute sums of Banach spaces," Journal of Mathematical Analysis and Applications, vol. 375, no. 1, pp. 207-222, 2011.

[12] K. Boyko, V. Kadets, M. Martín, and J. Merí, “Properties of lush spaces and applications to Banach spaces with numerical index 1," Studia Mathematica, vol. 190, no. 2, pp. 117-133, 2009. 


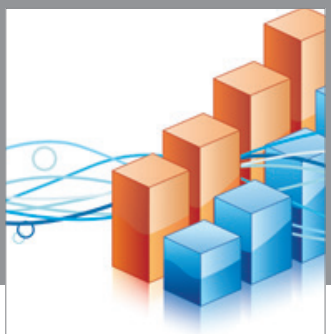

Advances in

Operations Research

vatem alat4

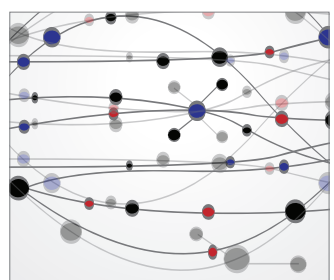

\section{The Scientific} World Journal
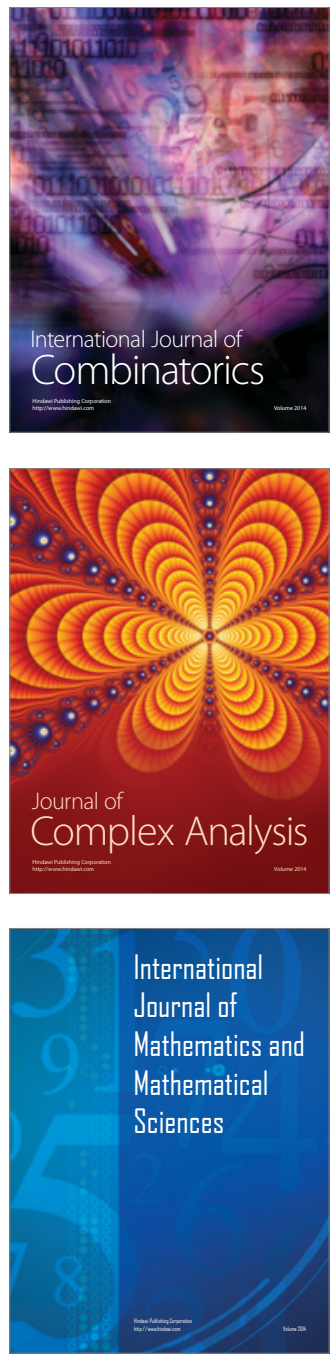
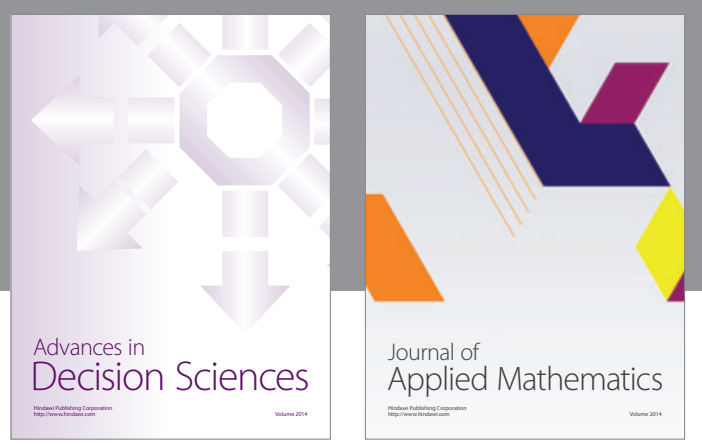

Algebra

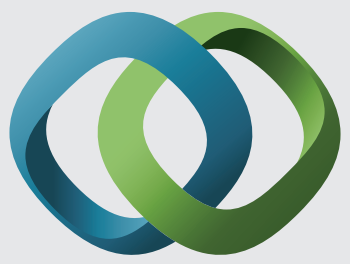

\section{Hindawi}

Submit your manuscripts at

http://www.hindawi.com
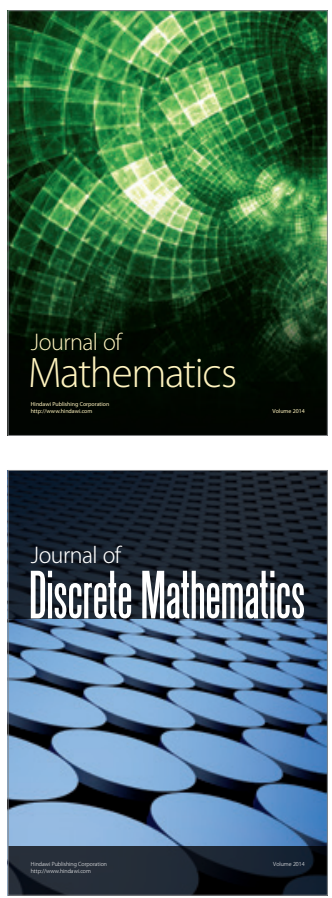

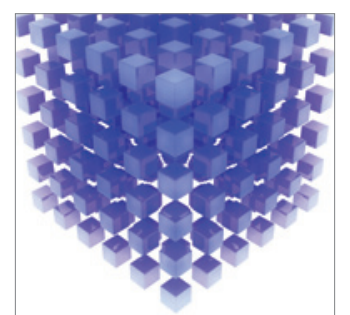

Mathematical Problems in Engineering
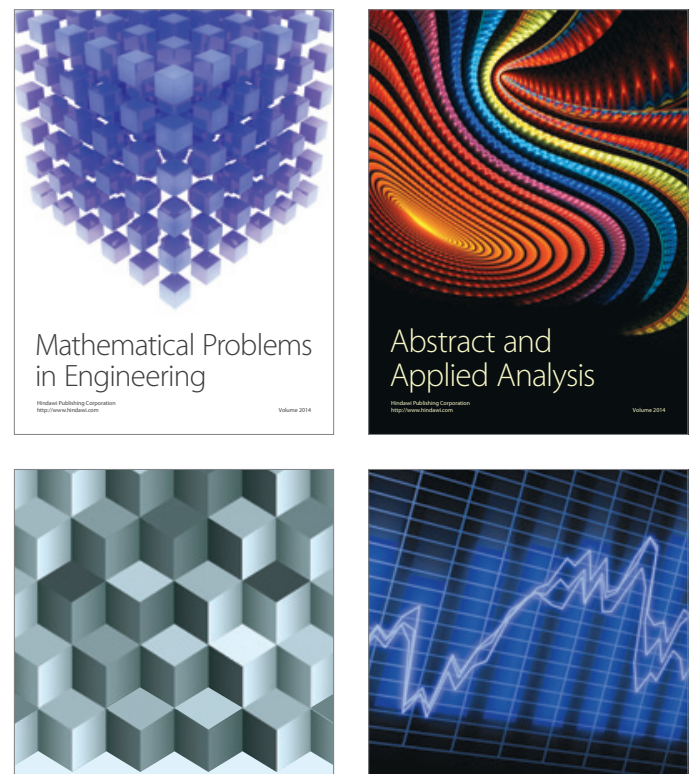

Journal of

Function Spaces

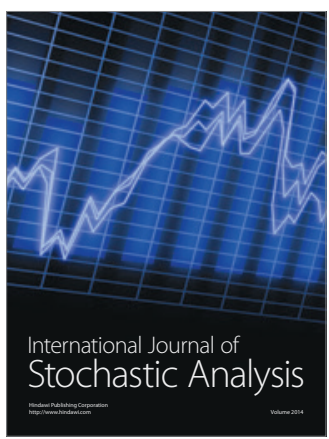

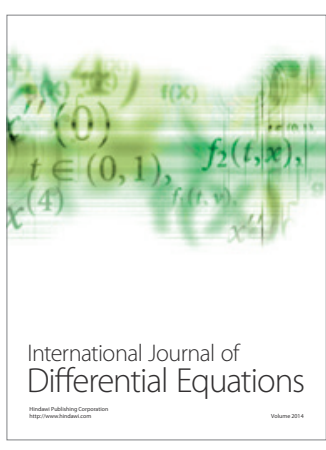
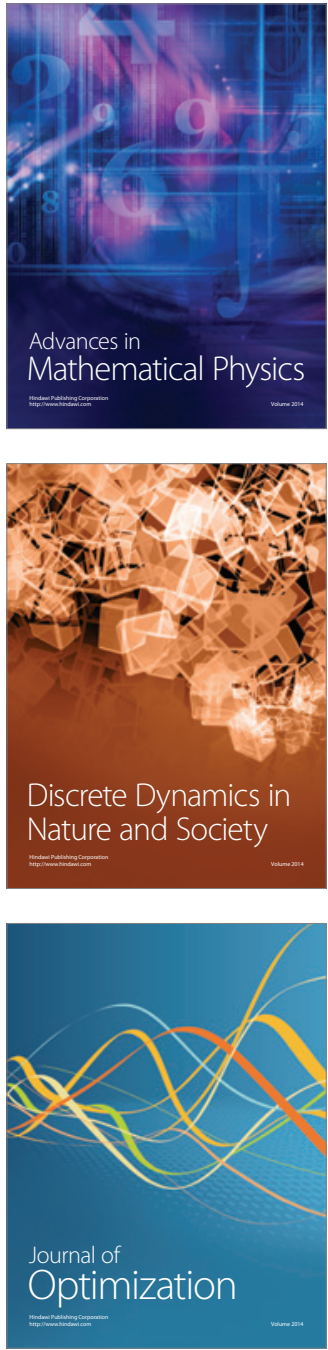